\title{
ANÁLISIS DE LAS \\ PRÁCTICAS Y ACCIONES \\ DE RESPONSABILIDAD \\ SOCIAL; UN ESTUDIO \\ DE CASOS EN LA \\ CORPORACIÓN CUN \\ REGIONAL MAGDALENA
}

\section{RESUMEN}

El proyecto analiza las políticas, prácticas y planes de responsabilidad social de la Corporación Unificada de Educación Superior, regional Magdalena, en la actualidad. Esto, con el fin de promover la memoria empresarial de prácticas relacionadas con la RSE, por medio de un modelo de medición de indicadores basado en ocho stakelholders relacionados con las variables de interés económico, social y ambiental. El análisis busca, además, medir la correlación que existe entre prácticas ejecutadas de RSE y niveles de percepción de los stakeholders más influyentes de la corporación.

Palabras clave: Responsabilidad Social Empresarial; Stakeholder; Dimensiones de la RSE. 
DEVINSO JIMÉNEZ SIERRA*

\section{ANALYSIS OF SOCIAL RESPONSIBILITY PRACTICES AND ACTIONS. A CASE STUDY IN CUN CORPORATION. MAGDALENA REGION}

\section{ABSTRACT}

The project will analyze the policies, practices and social responsibility plans of the Unified Regional Higher Education Corporation Magdalena today, in order to promote corporate memory related to CSR through a measurement model based on eight indicators related to changing economic, social and environmental stakelholders practices. The analysis also seeks to measure the correlation between CSR practices implemented and perceived levels of the most influential stakeholders of the corporation.

Keywords: Social Responsibility; Stakeholders; RSE dimensions.

\section{INTRODUCCIÓN}

Como herramienta que propicia altos estándares de competitividad a niveles internacionales, la Responsabilidad Social Empresarial, en adelante RSE, y la Responsabilidad Social Universitaria, en adelante RSU, se presentan como estrategias de gestión e innovación en el ámbito de las organizaciones, que buscan un mayor desarrollo social amparado en el concepto de sostenibilidad. Aunque en décadas pasadas se relacionó la RSE con una especie de filantropía que surgía como iniciativa de los empresarios de modo casual, desde principios del siglo XXI se determinó que la RSE podría convertirse en un elemento diferenciador y competitivo, que apunta a mejorar los procesos de reputación de la empresa, pensada más como un marketing social (Porter y Kramer, 2002).

Los análisis de RSE aplicados a instituciones de educación superior no son nuevos. Ya desde el año 2007, la Universidad Javeriana estructuró lo que se Ilamó el Observatorio de Responsabilidad Social Universitaria como un ente de verificación y apoyo al cumplimiento de los axiomas máximos de la ética aplicada a la formación integral de sus educandos. "Resulta cada vez más aceptado en el mundo académico, casi como una suerte de axioma, que la responsabilidad social de la Educación Superior y, en concreto, de la Universidad, es algo que pertenece a su misma naturaleza" (Remolina, 2007, p. 1). Del mismo modo, la Declaración Mundial sobre la Educación Superior en el siglo XxI: Visión y Acción, organizada por la Unesco y realizada en París del 5 al 9 de octubre de 1998, ha querido subrayar y reforzar este hecho perenne de la responsabilidad universitaria. Además, debe entenderse que la RSU va más allá del enfoque de la "proyección social y extensión universi-

\footnotetext{
* Magíster (c), docente tiempo completo Corporación Unificada Nacional de Educación Superior Cun, regional Magdalena. Colombia. Correo-e: devinso_jimenez@cun.edu.co.

Recibido: 10 de diciembre de 2015, aceptado: 3 de marzo de 2016.

Para citar el artículo: Jiménez, D. (2016). "Análisis de las prácticas y acciones de responsabilidad social; un estudio de casos en la corporación Cun, regional Magdalena", en Sotavento MBA, n. ${ }^{\circ} 27$, pp. 94-110. D0I: http://dx.doi.
} org/10.18601/01233734.n27.07 
taria" y apuesta por una reflexión integral de la universidad como institución académica que influye en el entorno social (Vallaeys, s.f., p. 3).

\section{FUNDAMENTO TEÓRICO}

En su forma básica, la RSE se refiere a la idea de que, en particular, las grandes organizaciones del sector empresarial, debido a su mayor potencia y efectos en las sociedades de todo el mundo (Neergaard, 2006), deberían disponer de un conjunto más amplio de responsabilidades hacia la sociedad en general, que van más allá del mero interés económico en términos legales y en general a las obligaciones de la empresa (Carroll, 1991). Aunque la RSE tradicionalmente se ha referido a las grandes empresas, el concepto se aplica a todas las empresas y organizaciones, y se ha convertido en un reto importante para cualquier empresa independientemente de su tamaño (Neergaard, 2006).

Tradicionalmente, la empresa fue considerada socialmente responsable de cumplir con las expectativas de la sociedad, contribuyendo a la economía en general y la creación de riqueza para la sociedad. Al principio, la función y responsabilidad de la empresa fue producir bienes y servicios, proporcionar puestos de trabajo y ser rentable por cualquier medio posible, independientemente de las consecuencias humanas y ambientales, siempre y cuando estuviese dentro del ámbito de la ley (Idowu, Filho, 2009).

En consonancia con esta visión básica sobre la empresa y sus responsabilidades, el muy citado economista estadounidense Milton Friedman (1970) argumenta que la única responsabilidad social de las empresas es aumentar los beneficios para sus propietarios 0 accionistas conforme a las reglas básicas de la sociedad en términos de cumplimiento de la ley y siguiendo las costumbres éticas actuales (Friedman, 1970). Según él, actividades sociales en términos de donación de dinero a buenas causas y hacer frente a otros problemas sociales son consideradas como una pérdida de "dinero y una distracción del propósito fundamental de una sociedad" (Friedman, 1970, p. 69).

Este punto de vista clásico de la empresa y sus responsabilidades es ampliamente citado como un claro contraste con las tendencias contemporáneas de la RSE. Sin embargo, todavía desempeña un papel en la definición de RSE, ya que forma la base de conceptualizaciones contemporáneas y la comprensión de la RSE.

La literatura contemporánea al respecto considera a las empresas responsables de un amplio grupo de intereses más allá de los propietarios o accionistas de la empresa. Esta concepción se basa en la teoría de la gestión de intereses, que define al interesado como cualquier grupo o individuo que puede afectar o es afectado por los logros de los objetivos de la empresa con el fin de obtener ganancias y mantenerse competitivos con altos estándares de calidad.

Las empresas deben identificar y actuar en acuerdo con las demandas y expectativas de sus grupos de interés (consumidores, empleados, inversores, gobiernos, proveedores, miembros de las comunidades locales, ONG, etc.) (Neergaard, 2006) y, en este sentido, la responsabilidad de una empresa se define por sus grupos de interés.

De acuerdo con la visión más amplia de la RSE, las empresas son una parte integral de la sociedad en la que operan. Sus acciones afectan a la sociedad en general y, por lo tanto, son consideradas responsables de sus impactos en la sociedad en su conjunto. Las empresas deben servir de manera constructiva a las necesidades de la sociedad, a satisfacción de la sociedad.

A fin de obtener certificaciones internacionales, como la norma Iso-26001 de responsabilidad social, que amplíen sus procesos de participación en mercados altamente exigentes, cuando se habla de las responsabilidades de las empresas para con la sociedad, se incluyen tanto los grupos de interés en su sentido origi- 
nal (propietarios o accionistas), como el medio ambiente, la riqueza general y la calidad de vida en las comunidades insertadas en el contexto geográfico en el que operan las empresas.

La Unión Europea (2001, p.6) define la RSE como: "Un concepto mediante el cual las empresas integran las preocupaciones sociales y medioambientales en sus negocios, operaciones y en sus interacciones con sus grupos de interés de manera voluntaria".

La Unión Europea especifica las preocupaciones sociales y ambientales como las principales áreas de responsabilidad a las que hacer frente de una forma voluntaria, después de su finalidad económica. RSE se refiere a la decisión voluntaria de las empresas de contribuir en la construcción de una sociedad mejor y un medioambiente más limpio (Comisión Europea, 2001, p. 4) por medio de la gestión, medición e impactos en la sociedad dentro de los ámbitos económico, social y ambiental.

Un elemento importante que no puedo dejar suelto es que la RSE se basa en un diálogo con las partes interesadas que, entre otras cosas, podría incluir alianzas con diversas instituciones, tales como sindicatos, autoridades públicas, fundaciones, centros de pensamiento, ONG, y representantes de negocios y organizaciones. El concepto de diálogo dentro de la perspectiva de una empresa omnipotente genera un alto nivel de ambigüedad pero, en realidad, la RSE busca establecer una relación cercana de sostenibilidad y eficiencia en respuesta al desgastado modelo de consumo continuo sin techo ni recursos necesarios para sostenerse.

La globalización no puede visionarse solo como una extensión o ampliación de mercados, sino como la implementación de una nueva tendencia global que obliga a las organizaciones empresariales a percibir el mundo de modo distinto. La difusión de la RSU debe ocupar, en el plano nacional, regional y local, gran parte de la agenda de competitividad de nuestras empresas. Si a nivel internacional la Unión
Europea ha establecido modelos de eficiencia empresarial, ampliamente difundidos en su libro verde, por medio de la concientización de una política empresarial más social, nuestras empresas deberían estar enfocadas en revisar y construir sus planes de responsabilidad social empresarial con miras a participar de esta nueva y necesitada visión de la sostenibilidad empresarial (Held, 2002).

\section{METODOLOGÍA}

La investigación es de tipo descriptivo. Se estableció una medición de la RSE tomando como referencia el modelo de indicadores de la RScat, realizando un ajuste y contextualización al modelo. El enfoque de la investigación es cualitativo, con herramientas de investigación cuantitativas.

En una primera fase, se miden el nivel de penetración de los valores, la percepción y conocimiento en RSE. Aquí se aplicó un instrumento fijado en 13 indicadores de clase sub-cero para establecer un primer nivel de medición. En una segunda fase, se midió el nivel de penetración de siete stakeholders con 225 indicadores de clase uno, con el siguiente nivel de participación:

Tabla 1. Grupos de interés objeto del estudio

\begin{tabular}{|c|c|c|c|}
\hline & $\begin{array}{l}\text { Grupos de Interés } \\
\text { (stakeholders) }\end{array}$ & \# Ind. & Participación \\
\hline 1 & Cliente interno & 78 & $35 \%$ \\
\hline 2 & Cliente externo & 33 & $15 \%$ \\
\hline 3 & Medioambiente & 37 & $16 \%$ \\
\hline 4 & Comunidad & 27 & $12 \%$ \\
\hline 5 & Gobierno & 18 & $8 \%$ \\
\hline 6 & Proveedores & 23 & $10 \%$ \\
\hline \multirow[t]{2}{*}{7} & Competencia & 9 & $4 \%$ \\
\hline & Número de indicadores & 225 & \\
\hline
\end{tabular}

Fuente: Elaboración propia. 
En una tercera fase, se aplicó un árbol de objetivo para escoger el grupo de interés de mayor impacto y realizar una medición de percepción de la RSE para luego contrastarlo con los niveles de penetración del mismo grupo de interés derivado de la fase 2 de este estudio. El stakeholder que se escogió fue el de cliente externo. Para ello, se tomó un muestreo aleatorio simple para una población finita de 1.956 clientes externos (estudiantes) (Fuente: coordinación académica) con un margen de error de $3 \%$, nivel de confianza de $95 \%$, y nivel de heterogeneidad de $50 \%$ para una muestra de 335 unidades de estudio.
Tabla 2. Proceso de investigación

\begin{tabular}{|c|c|c|c|}
\hline 1 & $\begin{array}{l}\text { Fase } 1 \\
\text { Nivel de penetación } \\
\text { de valores de RSE }\end{array}$ & $\begin{array}{l}13 \text { indicadores } \\
\text { de clase } 0\end{array}$ & $\begin{array}{l}\text { Valores } \\
\text { RSE }\end{array}$ \\
\hline \multirow[t]{2}{*}{2} & $\begin{array}{l}\text { Fase } 2 \\
\text { Nivel de penetración } \\
\text { de las acciones RSE }\end{array}$ & \multirow[t]{2}{*}{$\begin{array}{l}225 \text { indicadores } \\
\text { de clase } 1\end{array}$} & \multirow[t]{2}{*}{$\begin{array}{l}\text { Acciones } \\
\text { RSE }\end{array}$} \\
\hline & 7 Stakeholders & & \\
\hline 3 & $\begin{array}{l}\text { Fase } 3 \\
\text { Nivel de percepción } \\
\text { un stakeholders }\end{array}$ & $\begin{array}{l}33 \text { indicadores } \\
\text { de clase } 1\end{array}$ & $\begin{array}{l}\text { Percepción } \\
\text { de RSE }\end{array}$ \\
\hline
\end{tabular}

Fuente: Elaboración propia.

Tabla 3. Matriz de consistencia

\begin{tabular}{|c|c|c|c|c|}
\hline Tema & Problemas & Objetivos & Hipótesis & Variables \\
\hline \multirow{3}{*}{$\begin{array}{l}\text { Análisis de las } \\
\text { políticas, prácti- } \\
\text { cas y planes de } \\
\text { responsabilidad } \\
\text { social de la cun } \\
\text { Regional Costa } \\
\text { Caribe }\end{array}$} & $\begin{array}{l}\text { General } \\
\text { ¿Es la calidad de la prestación de } \\
\text { los servicios un fenómeno social que } \\
\text { afecta a todas las clases sociales } \\
\text { desde las familias más prestantes } \\
\text { hasta las más pobres, afirmando } \\
\text { que en los últimos años las personas } \\
\text { viven inconformes por la mala } \\
\text { prestación de servicio y por no } \\
\text { poder transitar cómodamente por } \\
\text { algunas calles? }\end{array}$ & $\begin{array}{l}\text { General } \\
\text { Identificar las prácticas, } \\
\text { planes y políticas de RSE que } \\
\text { tienen más impacto para la } \\
\text { Corporación Unificada de } \\
\text { Educación Superior. Regional } \\
\text { Costa Caribe, con el fin de } \\
\text { establecer indicadores de } \\
\text { gestión respecto de la RSE. }\end{array}$ & $\begin{array}{l}\text { Hipótesis general } \\
\text { Si las prácticas de RSE son } \\
\text { coherentes con las políticas } \\
\text { de RSE, entonces los niveles de } \\
\text { RSE son satisfactorios }\end{array}$ & $\begin{array}{l}\text { Variable independiente } \\
(\mathrm{X}) \\
\text { Factores internos de } \\
\text { la RSE } \\
\text { Variable dependiente } \\
\text { Términos de valoración } \\
\text { de la RSE. }\end{array}$ \\
\hline & $\begin{array}{l}\text { Específico } 1 \\
\text { Los aspectos relevantes a la RSE } \\
\text { están enmarcados en tres ejes } \\
\text { discrecionales: lo económico, lo } \\
\text { social y lo ambiental. Por ello, debe } \\
\text { terminarse en la Corporación su } \\
\text { nivel de injerencia }\end{array}$ & $\begin{array}{l}\text { Específico } 1 \\
\text { Determinar, por medio de } \\
\text { distintos instrumentos } \\
\text { de medición, el nivel de } \\
\text { penetración de los valores } \\
\text { de RSE de la Corporación } \\
\text { Unificada de Educación } \\
\text { Superior. Regional Costa } \\
\text { Caribe, teniendo como } \\
\text { variables, los componentes } \\
\text { económico, social y ambiental } \\
\text { de los diferentes stakeholders }\end{array}$ & $\begin{array}{l}\text { Hipótesis específica } 1 \\
\text { Si las prácticas de RSE son } \\
\text { coherentes con las políticas } \\
\text { de RSE, entonces los niveles } \\
\text { de RSE son satisfactorios }\end{array}$ & $\begin{array}{l}\text { Variable independiente } \\
\text { (X1) } \\
\text { Proceso de gestión de } \\
\text { calidad } \\
\text { Variable dependiente } \\
\text { (Y1) } \\
\text { Conocimiento de } \\
\text { aspectos generales de } \\
\text { RSE. }\end{array}$ \\
\hline & $\begin{array}{l}\text { Específico } 2 \\
\text { Una de las deficiencias en el manejo } \\
\text { de la RSE es la pobre discusión de } \\
\text { los términos relacionados con la } \\
\text { RSE, en muchos casos, los grupos } \\
\text { de interés que impactan a la } \\
\text { cooperación no conocen su papel } \\
\text { y/o rol dentro de la valoración de } \\
\text { las RSE. }\end{array}$ & $\begin{array}{l}\text { Específico } 2 \\
\text { Caracterizar los diferentes } \\
\text { stakeholders de la Corporación } \\
\text { Unificada de Educación } \\
\text { Superior. Regional Costa Caribe } \\
\text { que están involucrados en la } \\
\text { RSE de la misma desde las } \\
\text { variables económicas, sociales } \\
\text { y ambientales. }\end{array}$ & $\begin{array}{l}\text { Hipótesis específica } 2 \\
\text { Si las prácticas de RSE son } \\
\text { coherentes con las políticas } \\
\text { de RSE, entonces los niveles } \\
\text { de RSE son satisfactorios }\end{array}$ & $\begin{array}{l}\text { Variable independiente } \\
\text { (X2) } \\
\text { Aplicación de } \\
\text { instrumentos de } \\
\text { medición de modo } \\
\text { eficiente } \\
\\
\text { Variable dependiente } \\
\text { (Y2) } \\
\text { Experiencias } \\
\text { significativas }\end{array}$ \\
\hline
\end{tabular}




\begin{tabular}{|c|c|c|c|c|}
\hline Tema & Problemas & Objetivos & Hipótesis & Variables \\
\hline $\begin{array}{l}\text { Análisis de las } \\
\text { políticas, prácti- } \\
\text { cas y planes de } \\
\text { responsabilidad } \\
\text { social de la cun } \\
\text { Regional Costa } \\
\text { Caribe }\end{array}$ & $\begin{array}{l}\text { Específico } 3 \\
\text { Si no existe claridad conceptual } \\
\text { frente a los valores de RSE, no se } \\
\text { puede definir un marco estratégico } \\
\text { para la proyección de indicadores } \\
\text { de RSE }\end{array}$ & $\begin{array}{l}\text { Específico } 3 \\
\text { Definir cuál de los stakeholders } \\
\text { tiene mayor relevancia } \\
\text { dentro de las políticas, planes } \\
\text { o prácticas de RSE de la } \\
\text { Corporación Unificada de } \\
\text { Educación Superior. Regional } \\
\text { Costa Caribe }\end{array}$ & $\begin{array}{l}\text { Hipótesis específica } 3 \\
\text { Si no se tiene claridad } \\
\text { sobre la importancia de } \\
\text { la RSE como elemento } \\
\text { diferenciador del ejercicio } \\
\text { académico-administrativo de } \\
\text { la Corporación Unificada de } \\
\text { Educación Superior. Regional } \\
\text { Costa Caribe, entonces no } \\
\text { se podrá establecer un plan } \\
\text { coherente de RSE }\end{array}$ & $\begin{array}{l}\text { Variable independiente } \\
\text { (X3) } \\
\text { Tipos de stakeholders } \\
\text { identificados en la } \\
\text { Corporación Unificada } \\
\text { de Educación Superior. } \\
\text { Regional Costa Caribe }\end{array}$ \\
\hline
\end{tabular}

\section{ASPECTOS DE VALIDEZY CONFIABILIDAD}

La encuesta se validó en función de la evidencia relacionada con el constructo (Campos, 2012). La validez de constructo "se refiere a qué tan exitosamente un instrumento representa y mide un concepto teórico", y "parte del grado en el que las mediciones del concepto proporcionadas por el instrumento se relacionan de manera consistente con otras mediciones de otros conceptos, de acuerdo con modelos e hipótesis derivadas teóricamente (que conciernen a los conceptos que se están midiendo" (Hernández, Fernández y Baptista, 2006, p. 282). El proceso de validación de la escala se hizo mediante análisis factorial por análisis de componentes principales, lo que redujo la versión original de diez dimensiones y 269 ítems a solo 7 dimensiones y 225 ítems. Por otro lado, la encuesta ha demostrado una confiabilidad suficiente. La confiabilidad de un instrumento se refiere "al grado en que su aplicación repetida al mismo sujeto u objeto produce resultados iguales" (Hernández, Fernández y Baptista, 2006, p. 282). En ese sentido, la escala alcanzó una confiabilidad de 0,812 , medida con el coeficiente alfa Cronbach (Campos, 2012).

\section{RESULTADOS}

\section{NIVEL DE PENETRACIÓN DE LA RSE}

Según el instrumento aplicado, el análisis muestra que existe un conocimiento significativo de
Ios valores éticos corporativos dentro de CUN Regional Costa Caribe. Los indicadores que presentan mayor claridad son la identificación con el futuro deseado de la corporación, así como una identificación y proyección de la RSE en la construcción de la identidad estratégica. Otro aspecto importante es el conocimiento que se tiene, en la línea de dirección de la cun Regional Costa Caribe, sobre el significado de la RSE y su nivel de importancia y eje del desarrollo empresarial. Cabe anotar que los indicadores que menor nivel de penetración muestran son los relativos a los grupos de interés de la corporación, lo que va en contraposición con la conceptualización del significado de la RSE.

Otro aspecto de esta primera fase del estudio tiene que ver directamente con el conocimiento y manejo que tienen los colaboradores en general de la corporación respecto a la RSE, lo que indica que no existe una estrategia definida para orientar el discurso sobre la RSE a todos los niveles de la corporación. Ello está relacionado con los indicadores i8 e i9 sobre la socialización de los códigos de conducta a todos los clientes internos de la corporación, lo que pondría en riesgo el clima organizacional y los procesos de evaluación ponderados medianamente en este estudio.

Tabla 4. Matriz de puntuación ponderada de medición

\begin{tabular}{c|c}
\hline Puntuación & Nivel de penetración de la RSE \\
\hline $45-60$ & Altamente significativa \\
$21-44$ & Significativa \\
$01-20$ & Poco significativa \\
\hline
\end{tabular}

Fuente: Elaboración propia. 
Tabla 5. Indicadores de nivel de penetración de valores de RSE

\begin{tabular}{c|l|c}
\hline & \multicolumn{1}{|c|}{ Indicadores } & Resultado/60 \\
\hline i1 & Identidad estratégica y el futuro deseado institucional de la corporación. & 60 \\
\hline i2 & Disposición de códigos de ética o conducta formales. & 47 \\
\hline i3 & $\begin{array}{l}\text { Exposición pública de sus compromisos éticos por medio de materiales institucionales, por Internet } \\
\text { o de otra manera que sea adecuada a sus grupos de interés. }\end{array}$ & 49 \\
\hline i4 & Dignidad de la persona y valor estimado en los ámbitos de la corporación. & 46 \\
\hline i5 & $\begin{array}{l}\text { Coherencia entre los valores y principios éticos de la organización y la actitud individual de sus } \\
\text { empleados. }\end{array}$ & 49 \\
\hline i6 & Identidad estratégica y el futuro institucional en la responsabilidad social empresarial. & 60 \\
\hline i7 & Legislación vigente y aplicación responsable de la misma. & 48 \\
\hline i8 & Socialización de los códigos de ética o conducta entre los colaboradores de la corporación. & 41 \\
\hline i9 & $\begin{array}{l}\text { Cuestiones éticas en encuestas de ambiente organizativo, por evaluación 360º herramientas } \\
\text { similares. }\end{array}$ & 41 \\
\hline i10 & Conoce el significado de la RSE. & 54 \\
\hline i11 & Familiaridad con los temas relacionados con la RSE aplicándolos espontáneamente. & 38 \\
\hline i12 & Conoce los grupos de interés de la corporación. & 35 \\
\hline i13 & La Rse como una herramienta de desarrollo empresarial sostenible. & 50 \\
\hline
\end{tabular}

Fuente: Elaboración propia.

Este cuadro presenta la relación que se dio al analizar los niveles de penetración en cada uno de los indicadores propuestos con cada una de las unidades de análisis. Nos muestra una relación de las dimensiones que presentan menor puntaje, entre ellas, las que analizamos en el párrafo anterior -i8, i9, ill e i12-. Aunque no existe un indicador en un nivel poco significativo, es necesario establecer una medida de control frente a los indicadores de baja frecuencia.

Gráfica 1. Comportamiento por indicador de fase 1

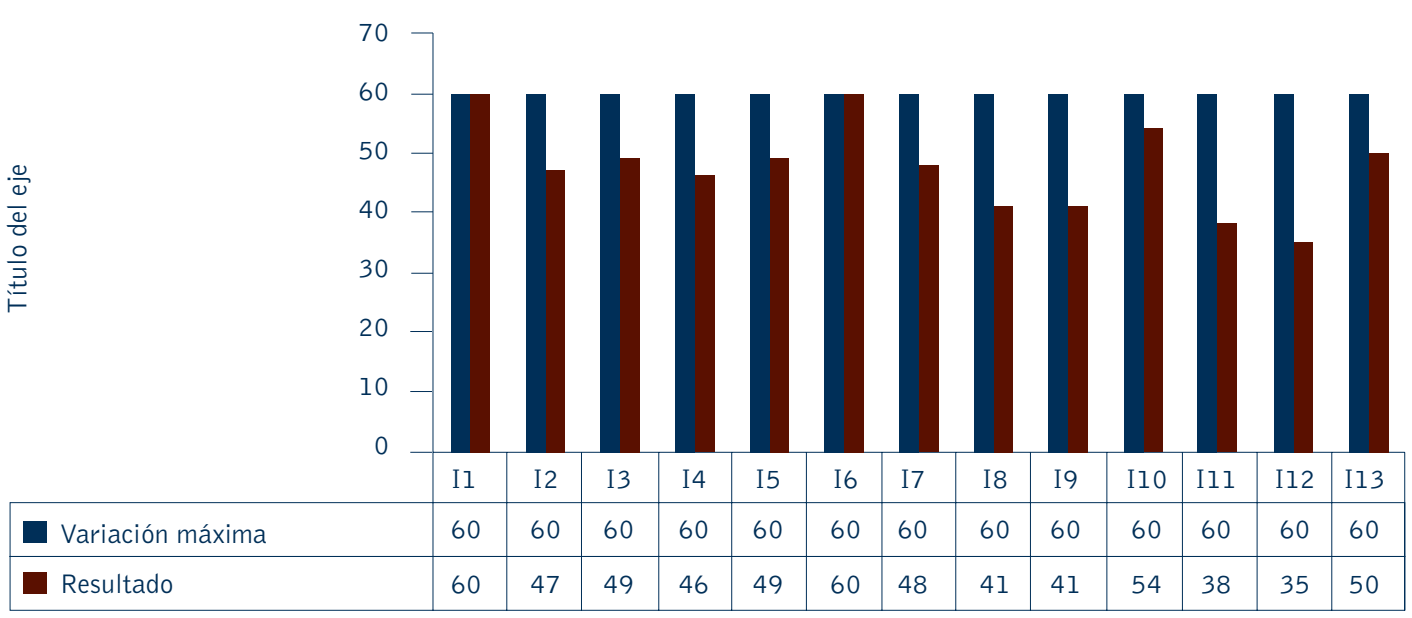

Fuente: Elaboración propia. 


\section{COMPORTAMIENTO DE STAKEHOLDERS}

\section{CLIENTE INTERNO}

En un primer análisis, se establecieron los niveles de incorporación de la RSE en la cUn Magdalena desde los stakeholders: cliente interno, cliente externo, comunidad, medioambiente, proveedores, gobierno y competencia. Para cada uno de ellos, se estableció una escala de significación y una tabla que midió ponderado de cada dimensión según el número de indicadores, promedio de la dimensión, la variación máxima por indicador, el puntaje de la dimensión y su respectiva variación máxima, y se fijó el nivel de significación por dimensión.

Tabla ponderada de puntuación aplicada para todas las escalas de medición de niveles de penetración de RSE

Tabla 6. Matriz de puntuación ponderada de medición

\begin{tabular}{c|c}
\hline Puntuación & Nivel de penetración de la RSE \\
\hline $3,34-5$ & Altamente significativa \\
\hline $1,7-3,33$ & Significativa \\
\hline $0-1,6$ & Incipiente \\
\hline
\end{tabular}

Fuente: Elaboración propia.

Tabla 7. Indicadores cliente interno

\begin{tabular}{c|l|c|c|l}
\hline & Dimensiones & \# Ind. & Promedio & Nivel \\
\hline 1 & Relaciones con asociaciones de empleados & 4 & 2,3 & Significativo \\
\hline 2 & Gestión participativa & 3 & 3,5 & Altamente Significativo \\
\hline 3 & Compromiso frente al primer empleo & 7 & 3,2 & Significativo \\
\hline 4 & Compromiso con el desarrollo infantil & 16 & 1,0 & Incipiente \\
\hline 5 & Valoración de la diversidad & 8 & 3,4 & Significativo \\
\hline 6 & Compromiso con la promoción de la equidad de género & 8 & 3,6 & Altamente Significativo \\
\hline 7 & Política de remuneración, prestaciones y carrera & 9 & 3,1 & Significativo \\
\hline 8 & Cuidados de salud, seguridad y condiciones de trabajo & 10 & 3,1 & Significativo \\
\hline 9 & Compromiso con el desarrollo profesional y la empleabilidad & 9 & 2,0 & Incipiente \\
\hline 10 & Conducta frente a despidos & 4 & 3,4 & Significativo \\
\hline & Número de indicadores & 78 & 2,86 & \\
\hline
\end{tabular}

Fuente: Elaboración propia.

En esta tabla, después de haber analizado 78 indicadores discriminados en 10 dimensiones de la variable stakeholder cliente interno, se estableció que existen 2 dimensiones en un estado de significación incipiente, entre ellos, la dimensión que respecta a los compromisos con el desarrollo infantil con 16 indicadores que muestra un ponderado de 15,5 puntos frente a su variación máxima de 50 puntos, lo que representa un proporción del $34 \%$ de cumplimiento. Dentro de los indicadores más críticos (ver anexo, cuadro de indicadores cliente interno) están los tendientes al apoyo flexible de los horarios a madres lactantes, campañas de inclusión de los hijos de los empleados en la escuela, así como el indicador i28 sobre el control y seguimiento a los hijos menores de 5 años de los trabajadores de la institución (ver indicadores de i15 a i30). La siguiente dimensión crítica es la número 9, sobre los compromisos con el desarrollo profesional y la empleabilidad, contenida en 9 indicadores de los cuales preocupa la falta de evaluación a la rotación de la planta de trabajadores (i67), y el hecho de no considerar la situación socioeconómica del empleado al momento de su despido (i71), entre otros. 


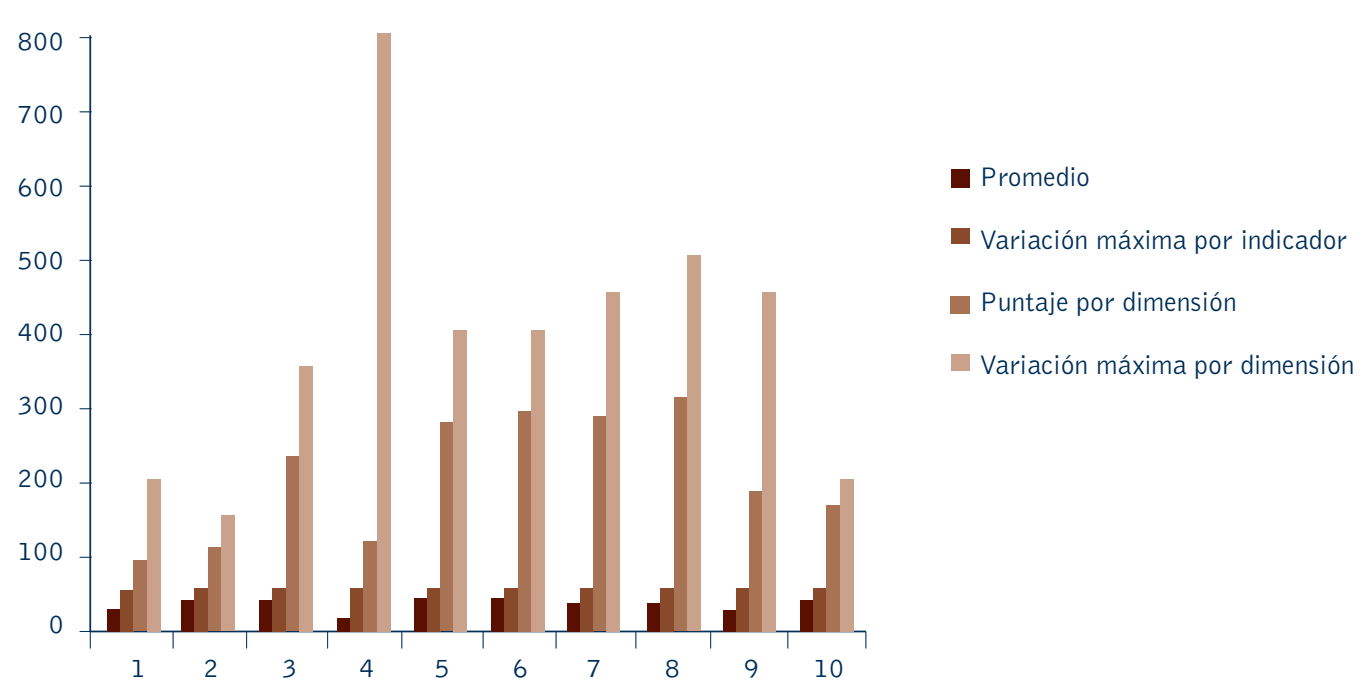

Fuente: Elaboración propia.

Esta gráfica muestra el comportamiento de cada dimensión según los criterios: promedio de cada dimensión, variación máxima por indicador, puntaje por dimensión y su variación máxima. La gráfica sustenta de mejor modo la relación desproporcionada de la dimensión número 4, que presenta un puntaje de 115 sobre una variación máxima de 800 puntos, lo que es un resultado crítico para el estudio.

\section{CLIENTE EXTERNO}

Al contrastar las categorías de escala por dimensión de la variable cliente externo compuesta por (5) cinco dimensiones y 33 indicadores constatamos que esta variable es la que mejor comportamiento presenta al no registrar dimensiones en grado incipiente, aunque en la dimensión número (2) se presentan con puntaje crítico dos dimensiones i7 sobre la existencia de un defensor del servicio o con funciones similares, que presenta un ponderado de 20,1 sobre una variación máxima de 50 puntos.
Tabla 8. Indicadores cliente interno

\begin{tabular}{|c|c|c|c|c|}
\hline & Dimensiones & $\begin{array}{l}\# \\
\text { ind. }\end{array}$ & Promedio & Nivel \\
\hline 1 & Orientación al cliente & 4 & 4,1 & $\begin{array}{l}\text { Altamente } \\
\text { significativo }\end{array}$ \\
\hline 2 & $\begin{array}{l}\text { Marketing responsable } \\
\text { direccionado a los } \\
\text { clientes }\end{array}$ & 5 & 3,1 & Significativo \\
\hline 3 & $\begin{array}{l}\text { Clientes indirectos } \\
\text { o subclientes (niños, } \\
\text { adolescentes) }\end{array}$ & 3 & 3 & Significativo \\
\hline 4 & $\begin{array}{l}\text { Excelencia en la } \\
\text { atención }\end{array}$ & 16 & 3,8 & $\begin{array}{l}\text { Altamente } \\
\text { significativo }\end{array}$ \\
\hline 5 & $\begin{array}{l}\text { Conocimiento y } \\
\text { gerenciamiento de los } \\
\text { daños potenciales de } \\
\text { los servicios }\end{array}$ & 5 & 3,9 & $\begin{array}{l}\text { Altamente } \\
\text { significativo }\end{array}$ \\
\hline & $\begin{array}{l}\text { Número de } \\
\text { indicadores }\end{array}$ & 33 & 3,58 & \\
\hline
\end{tabular}

Fuente: Elaboración propia. 
Gráfica 3. Comportamiento de significación grupo de interés cliente externo

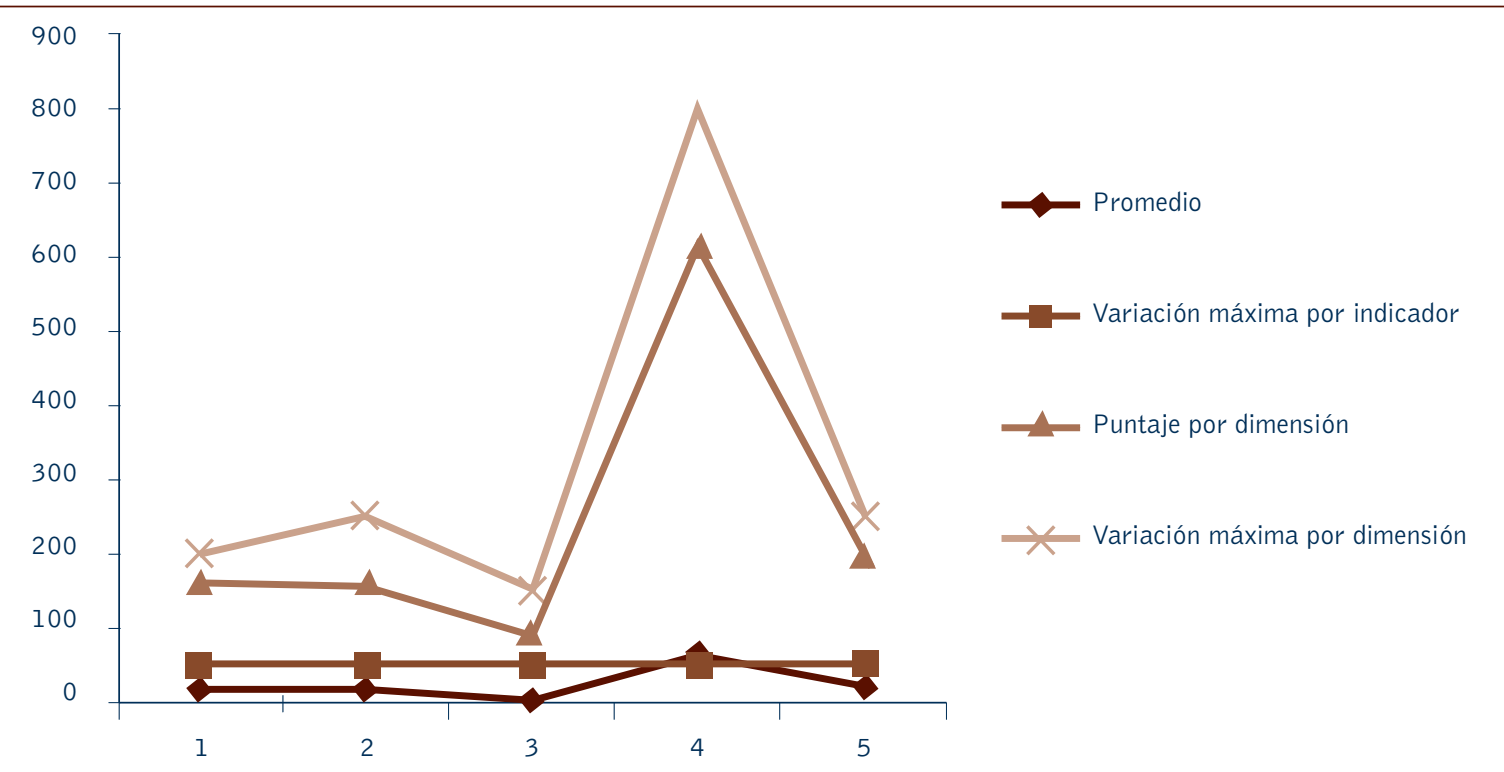

Fuente: Elaboración propia.

Analizando la tabla de categorías de escala y la gráfica de comportamiento general, Ilama la atención que la dimensión que más indicadores mide alcanza el nivel de significación más alto, lo que representa un aspecto positivo de la apropiación de la responsabilidad social empresarial de la cun Magdalena frente a la dimensión número 4, que mide el tema de la excelencia en la atención. Más adelante contrastaremos los niveles de apropiación con los niveles de percepción de esta variable de estudio.

\section{MEDIOAMBIENTE}

Este stakeholder presenta una línea de 5 dimensiones, que mide desde la política de gestión ambiental hasta los planes de mitigación de entradas y salidas de insumos. Dentro de los tres aspectos fundantes de la RSE se encuentran lo económico, lo social y lo ambiental, discriminados en esta variable. En estas (5) cinco dimensiones se midieron 37 indicadores, de los cuales solo uno alcanzó el nivel de significancia medio significativo; las otras (4) cuatro dimensiones presentan niveles de significación incipiente.

Tabla 9. Indicadores stakeholder medio ambiente

\begin{tabular}{l|l|c|c|c}
\hline \multicolumn{1}{|c|}{ Dimensiones } & $\begin{array}{c}\# \\
\text { ind. }\end{array}$ & Promedio & Nivel \\
\hline 1 & $\begin{array}{l}\text { Política y gestión } \\
\text { ambiental }\end{array}$ & 6 & 1,23 & Incipiente \\
\hline $\begin{array}{l}\text { Compromiso con el } \\
\text { mejoramiento de la } \\
\text { calidad ambiental }\end{array}$ & 6 & 1,32 & Incipiente \\
\hline 3 & $\begin{array}{l}\text { Educación y } \\
\text { concienciación } \\
\text { ambiental }\end{array}$ & 4 & 2,75 & Significativo \\
\hline 4 & $\begin{array}{l}\text { Gerenciamiento de } \\
\text { los impactos sobre } \\
\text { el medioambiente y } \\
\text { del ciclo de vida de } \\
\text { productos y servicios }\end{array}$ & 13 & 1,6 & Incipiente \\
\hline 5 & $\begin{array}{l}\text { Minimización de } \\
\text { entradas y salidas de } \\
\text { insumos }\end{array}$ & 8 & 1,6 & Incipiente \\
\hline & $\begin{array}{l}\text { Número de indicadores } \\
\text { Furas }\end{array}$ & 37 & & \\
\hline
\end{tabular}

Fuente: Elaboración propia.

La tabla anterior nos muestra el estado incipiente en que se encuentra el stakeholder 
medioambiente, preocupa el estado crítico de las dimensiones D1, D2 y D3, que presentan puntuaciones por debajo del $25 \%$ de la variación máxima de cada dimensión, sobre todo la dimensión D4, con el mayor número de parti- cipación respecto a su número de indicadores (13 en total). Máxime si se trata de la dimensión sobre la gerencia de los impactos sobre el medioambiente y del ciclo de vida de los productos, efecto explícito en la siguiente gráfica.

Gráfica 4. Comportamiento medioambiente

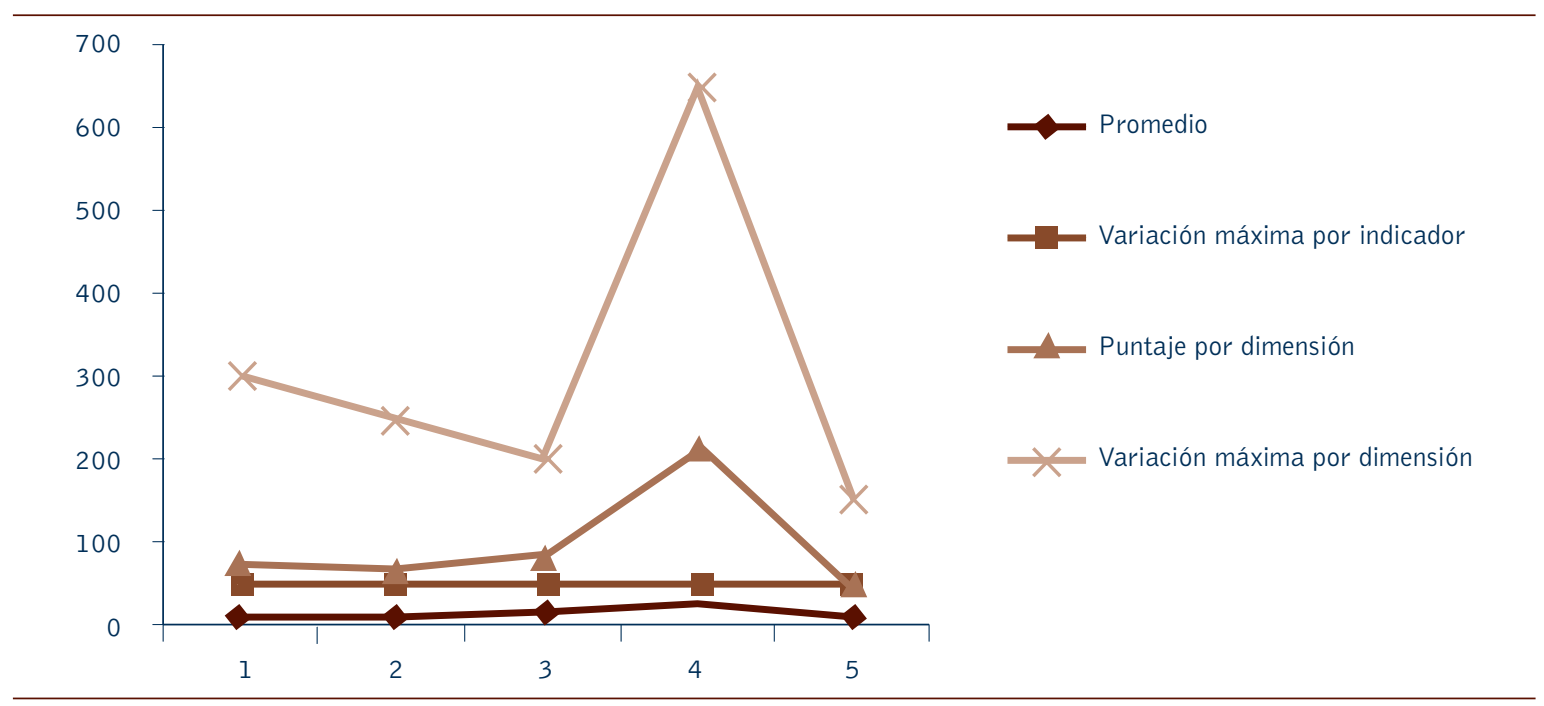

Fuente: Elaboración propia.

Respecto a la anterior gráfica de comportamiento, encontramos un pico de variación máxima de la dimensión: 650 puntos, muy lejos de la puntuación de la dimensión con mayor puntuación, 208 puntos.

\section{COMUNIDAD}

Este stakeholder mide 4 dimensiones relacionadas con la inclusión que tiene la corporación en la comunidad circundante; mide desde la participación de la comunidad en la empresa, hasta los compromisos con la acción social. Se resalta de esta variable de estudio los niveles de significación altos en 3 de las cuatro dimensiones.
Tabla 10. Indicadores stakeholder comunidad

\begin{tabular}{l|l|c|c|l}
\hline Dimensiones & $\begin{array}{c}\# \\
\text { ind. }\end{array}$ & Promedio & Nivel \\
\hline $\begin{array}{l}\text { Presencia en el mercado } \\
\text { y uso de los recursos } \\
\text { locales, favoreciendo el } \\
\text { desarrollo económico } \\
\text { local }\end{array}$ & 2 & 0,5 & Incipiente \\
\hline 2 & $\begin{array}{l}\text { Gerencia del impacto } \\
\text { de la corporación en la } \\
\text { comunidad de entorno }\end{array}$ & 13 & 3,85 & $\begin{array}{l}\text { Altamente } \\
\text { significativo }\end{array}$ \\
\hline 3 & $\begin{array}{l}\text { Financiamiento de la } \\
\text { acción social }\end{array}$ & 6 & 3,7 & $\begin{array}{l}\text { Altamente } \\
\text { significativo }\end{array}$ \\
\hline $\begin{array}{l}\text { Compromiso con la } \\
\text { acción social }\end{array}$ & 6 & 3,6 & $\begin{array}{l}\text { Altamente } \\
\text { significativo }\end{array}$ \\
\hline & Número de indicadores & 27 & 2,9 & \\
\hline
\end{tabular}

Fuente: Elaboración propia. 
La tabla anterior muestra un compromiso altamente significativo respecto a la valoración del grupo de interés comunidad. Este aspecto es relevante siempre que se entiende como uno de los tres principales pilares de la RSE el aspecto social representado altamente en la comunidad circundante y comunidad en general.
Sin embargo, una de las dimensiones presenta un nivel de significación incipiente. Esta es la dimensión D1: presencia en el mercado y uso de los recursos locales favoreciendo el desarrollo social económico, que solo alcanzó el $10 \%$ del total de su variación máxima, con 10 puntos sobre 100 posibles.

Gráfico 5. Indicadores stakeholder comunidad

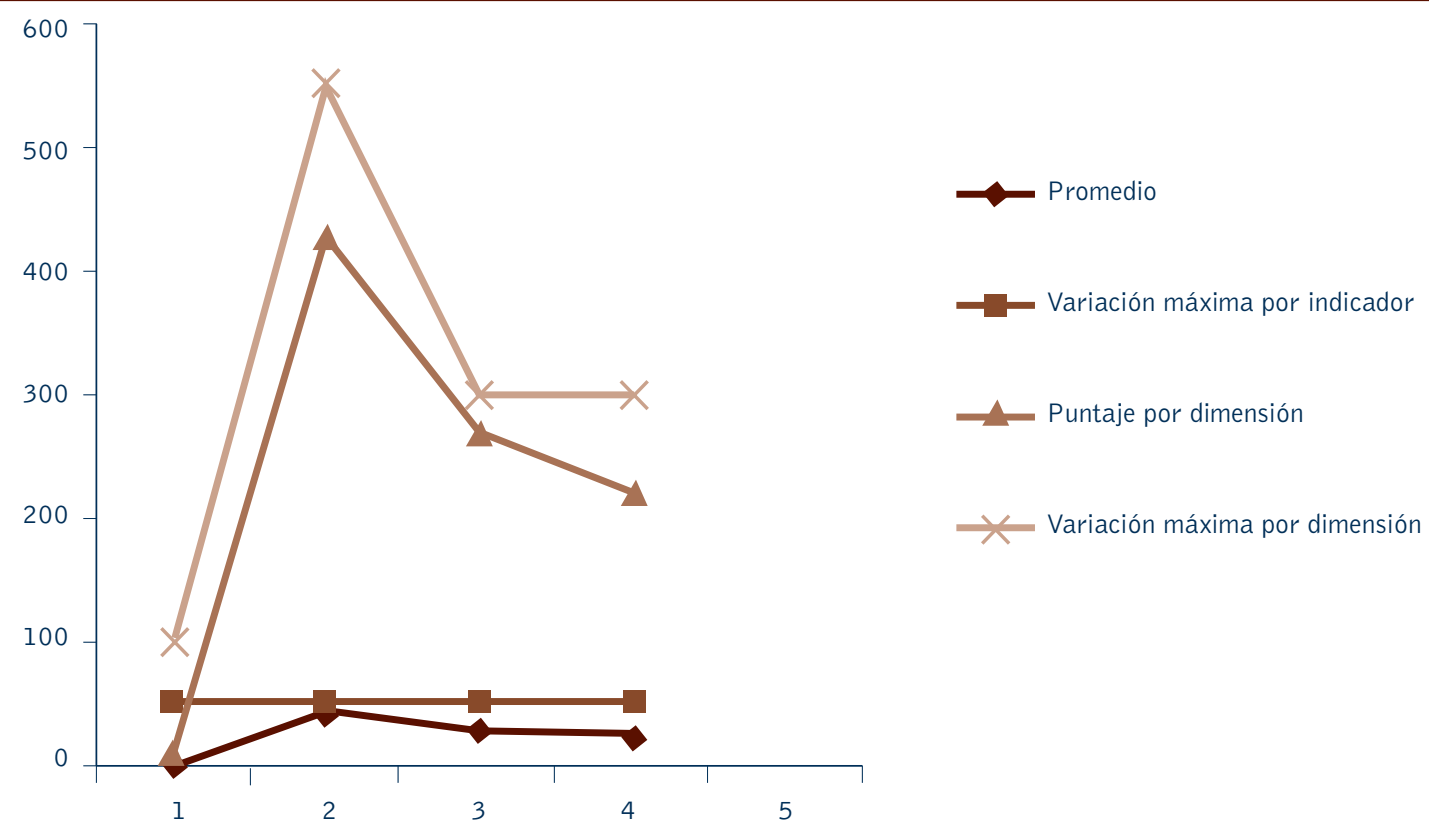

Fuente: Elaboración propia.

La gráfica ilustra el comportamiento de los puntajes obtenidos en cada dimensión y las variaciones máximas de cada una, mostrando una coincidencia considerable en la asimilación de la RSE por parte de este grupo.

\section{GOBIERNO}

Este grupo de interés representa la relación de la corporación con el sector externo, ante todo con los entes gubernamentales, tales como gobernación, alcaldía u ONG. En este aspecto, la cUn presenta unos resultados destacados, que son coherentes con las políticas de relacionamiento con el sector externo que tiene la corporación. Este stakeholder está compuesto por 4 dimensiones discriminadas en 20 indicadores. De las cuatro dimensiones, tres están en un nivel altamente significativo y una en nivel significativo, lo que representa un logro en el proceso de asimilación de la RSE desde la práctica misma, sin la existencia de una política que así lo exigiera. 
Tabla 11. Indicadores stakeholder gobierno

\begin{tabular}{l|l|l|l}
\hline \multicolumn{1}{c|}{ Dimensiones } & \multicolumn{1}{c|}{ \# ind. } & \multicolumn{1}{c}{ Promedio } & \multicolumn{1}{c}{ Nivel } \\
\hline Empresa/ciudadanía & 5 & 2,6 & Significativo \\
\hline Anticorrupción & 3 & 3,7 & Altamente significativo \\
\hline Liderazgo empresa/gobierno & 5 & 3,63 & Altamente significativo \\
\hline Participación en proyectos gubernamentales o municipales & 5 & 3,92 & Altamente significativo \\
\hline Número de indicadores & 18 & 3,4 & \\
\hline
\end{tabular}

Fuente: Elaboración propia.

Gráfico 6. Comportamiento indicadores stakeholder gobierno

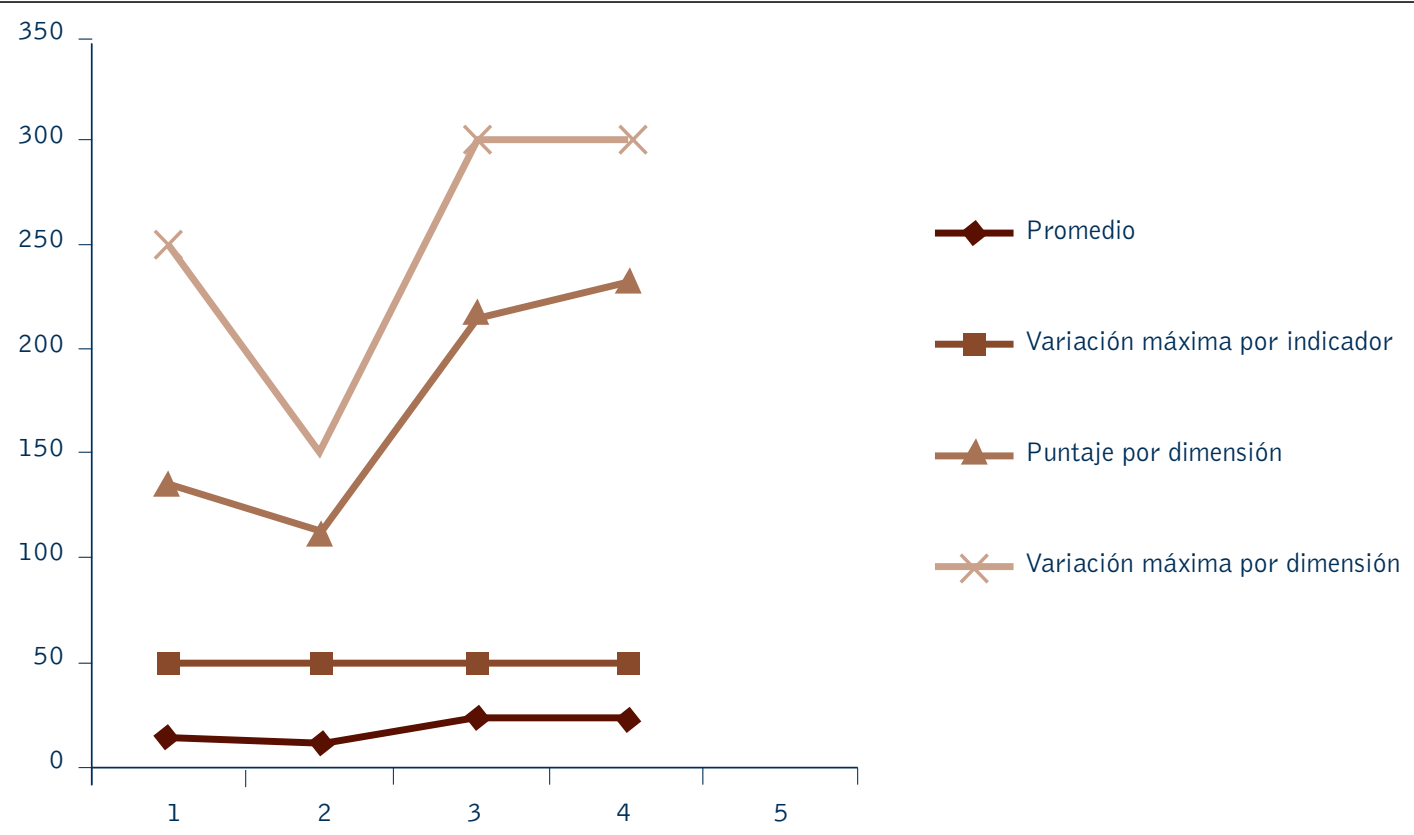

Fuente: Elaboración propia.

Las tablas y gráfica anteriores muestran los resultados por dimensión, la participación por nivel de significación y el comportamiento del puntaje obtenido por la variable frente a la variación máxima presentada por cada dimensión. Es de resaltar la apropiación de este grupo de interés dentro de las prácticas de RSE de la cun Costa Caribe.

\section{PROVEEDORES}

Esta dimensión mide tres indicadores: criterios de selección y evaluación de proveedores, apoyo al desarrollo de proveedores y relación de doble vía. Esta dimensión presenta dos indicadores en nivel de significación y uno en nivel altamente significativo.

Tabla 12. Indicadores stakeholder proveedores

\begin{tabular}{c|l|c|c|c}
\hline \multicolumn{1}{|c|}{ Dimensiones } & $\begin{array}{c}\# \\
\text { ind. }\end{array}$ & Promedio & Nivel \\
\hline 1 & $\begin{array}{l}\text { Criterios de selección } \\
\text { y evaluación de } \\
\text { proveedores }\end{array}$ & 11 & 3,2 & Significativo \\
\hline 2 & $\begin{array}{l}\text { Apoyo al desarrollo de } \\
\text { proveedores }\end{array}$ & 8 & 3,6 & $\begin{array}{l}\text { Altamente } \\
\text { Significativo }\end{array}$ \\
\hline 3 & Relaciones de doble vía & 4 & 3,4 & $\begin{array}{l}\text { Altamente } \\
\text { Significativo }\end{array}$ \\
\hline & Número de indicadores & 23 & 3,35 & \\
\hline
\end{tabular}

Fuente: Elaboración propia. 
De los 23 indicadores, vale mencionar que tres de ellos están en niveles incipientes, el i1, i2 e i7, todos dentro de la D1. Estos indicadores muestran la falta de una política clara de selección de proveedores en función del compromiso ético de los proveedores.

\section{COMPETENCIA}

Esta variable tiene una sola dimensión que busca medir por medio de 9 indicadores el nivel de relacionamiento que existe con la competencia, en este caso, con otras universidades de la región.

Tabla 13. Indicadores stakeholder competencia

\begin{tabular}{c|l|c|c|c}
\hline & \multicolumn{1}{|c|}{ Dimensiones } & $\begin{array}{c}\# \\
\text { ind. }\end{array}$ & Promedio & Nivel \\
\hline 1 & $\begin{array}{l}\text { Relaciones con la } \\
\text { competencia }\end{array}$ & 9 & 1,3 & Incipiente \\
\hline & Número de indicadores & 9 & 1,3 & \\
\hline
\end{tabular}

Fuente: Elaboración propia.

En esta variable, los resultados no son satisfactorios. A pesar de medir solo una dimensión, el nivel de significación es incipiente. Todos los indicadores tienen puntuación por debajo de 25, cuando su variación máxima es de 50 puntos. Si hablamos de la dimensión en general, su puntuación es de 120, cuando su variación máxima es de 450 puntos.

\section{RESUMEN: NIVEL DE PENETRACIÓN}

En esta segunda fase, el resultado de la medición del nivel de penetración es significativo bajo, con un promedio general de 2,6. Aunque existe un reconocimiento de la RSE y un manejo inicial de algunos indicadores de aplicación de RSE, las acciones no corresponden con el nivel más alto de significación de la RSE. Se evidencia, además, la incidencia de grupos de interés que no están siendo monitoreados, lo que no permite tener factor de medición frente a su impacto en la corporación. Ellos son los grupos de interés del medioambiente y el de la relación con la competencia. También hay que afirmar que los procesos de seguimiento, gestión y uso responsable del mercadeo se ven reflejados en un nivel altamente significativo de su grupo de interés: cliente externo y las relaciones con los entes gubernamentales.

Tabla 14. Resumen de indicadores de todos los stakeholders

\begin{tabular}{l|l|c|c|c|l}
\hline \multicolumn{1}{|c|}{ Grupos de interés } & \# Ind. & Participación & Promedio & \multicolumn{1}{c}{ Nivel } \\
\hline 1 & Cliente interno & 78 & $35 \%$ & 2,87 & Significativo \\
\hline 2 & Cliente externo & 33 & $15 \%$ & 3,58 & Altamente significativo \\
\hline 3 & Medioambiente & 37 & $16 \%$ & 1,6 & Incipiente \\
\hline 4 & Comunidad & 27 & $12 \%$ & 2,9 & Significativo \\
\hline 5 & Gobierno & 18 & $8 \%$ & 3,4 & Altamente significativo \\
\hline 6 & Proveedores & 23 & $10 \%$ & 3,2 & Significativo \\
\hline 7 & Competencia & 9 & $4 \%$ & 1,3 & Significativo \\
\hline & Número de indicadores & 225 & & 2,6 & \\
\hline
\end{tabular}

Fuente: Elaboración propia. 
Gráfico 7. Comportamiento de indicadores todos los stakeholders

Comportamiento general de cada Stakeholder

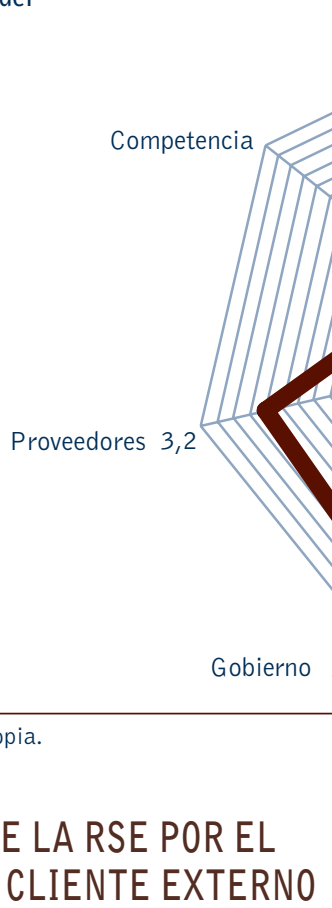

\section{PERCEPCIÓN DE LA RSE POR EL STAKEHOLDER CLIENTE EXTERNO}

La medición de los niveles de percepción de la RSE desde el grupo de interés cliente externo, que en este caso responde a nuestros estudiantes, muestra una variación respecto a los resultados de medición de los niveles de penetración y asimilación de la RSE dados en la fase 2. La variación es relevante en cuanto todas las dimensiones varían su nivel de significación en cada fase de estudio, tal como lo muestra la siguiente tabla. Mientras Ia D1 es altamente significativa en la fase de estudio 2, es percibida solo como significativa. La D2: mercadeo responsable direccionado a los clientes, que en el estudio de apropiación presenta un nivel significativo, es percibido como altamente significativo. Entre las variaciones más relevantes se encuentra la dada en la D5: conocimiento y gerenciamiento de los daños potenciales de los servicios, que en la medición de penetración y asimilación de la RSE se presenta en nivel altamente significativo, es percibido como incipiente.

Tabla 15. Contraste de indicadores de nivel de penetración vs. nivel percepción: stakeholder cliente externo

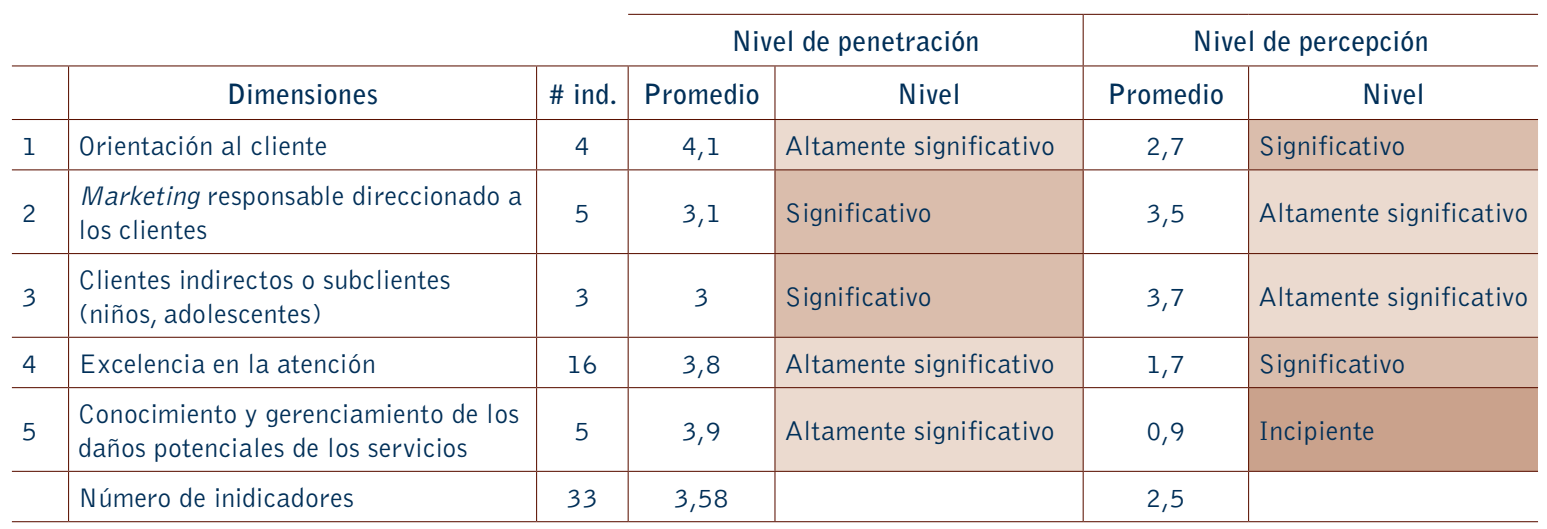

Fuente: Elaboración propia. 


\section{CONCLUSIONES Y DISCUSIÓN}

Para establecer los antecedentes del proyecto, es importante conocer algunas variaciones prácticas del marco conceptual de la RSE debido a que es posible que las prácticas corporativas atiendan particularmente a unos grupos de interés sin establecer como tal un ejercicio dinámico de la RSE. En la actualidad, nos encontramos inmersos en una sociedad cada vez más globalizada y uno de los alcances de esa globalización es que las consecuencias de nuestras acciones impactan cada vez a más personas en una cantidad cada vez más amplia de lugares. Cuando asumimos esta realidad, estamos ante lo que se conoce como Responsabilidad Social (Valdivia, 2007). Dentro de las conclusiones de la investigación tenemos:

- Tal como lo indican los resultados y consecuente con el objetivo específico propuesto, existe un nivel de penetración de la RSE que aunque se muestre significativo no es lo suficientemente dominante. Es decir, existe un conocimiento particular de la RSE pero no una apropiación de la misma por parte del personal administrativo, ejecutivo y gerencial de la corporación, ello debido a que no hay una política clara del manejo de la RSE y, por ende, no existen planes definidos de RSE dentro de la organización.

- Existe un desconocimiento de los grupos de interés que tiene afectación dentro de la corporación. No se entiende, por ejemplo, que la competencia es un grupo de interés para la organización, más allá de la medición de precios o de servicios. Además, se evidenció la poca coherencia de la organización con el gerenciamiento de los aspectos relacionados con el stakeholder medio ambiente, lo que no le permite estandarizar sus niveles de control y gerencia sobre planes de manejo ambiental. La caracterización muestra un descuido de varios grupos de interés que son importantes en el ámbito global para las organizaciones modernas.

- El stakeholder más relevante de la cun Magdalena es el del cliente externo, con un nivel de penetración y apropiación altamente significativo pero en detrimento de otros grupos de interés, como medioambiente, comunidad, competencia y proveedores, que tiene poca incidencia. El desarrollo teórico de la RSE como un conjunto de conceptos y marcos de referencia justificados, como un campo progresivo con fronteras disciplinares permeadas por los conceptos sociales de la Responsabilidad, presenta el concepto de RSE como un paradigma dominante. Tal como lo muestra el antecedente de este estudio, las investigaciones en este campo son una tendencia académica.

Dentro del estudio realizado, se presentan disposiciones de forma y de fondo. De forma, que la manera en que se establecen los indicadores puede variar según el enfoque que se pretenda en la investigación. En este caso, la investigación muestra los niveles de penetración, asimilación y percepción de la RSE desde la óptica organizacional, entendida la cun como un ente de gobierno corporativo, estimando su función y dinamismo a estándares internacionales corporativos, pero podría pensarse en un enfoque desde la RSU (Responsabilidad Social Universitaria) que en este caso no es el objeto de estudio. De fondo, esta visión de la RSE en la CUN Magdalena muestra las prácticas habituales en las que aplicamos principios de responsabilidad social, pero esta no está a favor de la cadena de valor de la corporación. No se encauzan las actividades de salida con las actividades de apoyo (Porter, 1985), lo que no nos permite tener planes de manejo para direccionar los esfuerzos económicos en función de la generación de valor social y el control y gerenciamiento del valor ambiental. 
La investigación muestra la necesidad de establecer una herramienta que nos permita medir periódicamente los esfuerzos de la organización a favor de la generación de valor económico, valor social y valor ambiental, de tal manera que podamos hacer control y gerencia de la RSE. No pueden existir planes de responsabilidad social si no hay conocimiento, apropiación, dominio de la misma; no se puede ejercer una gerencia eficiente si no se tiene una política definida sobre la RSE que permita encauzar la administración y gestión de los stakeholders relacionados con la corporación.

\section{REFERENCIAS}

Carroll, A. (1991): "La pirámide de la Responsabilidad Social Empresarial: hacia la moral en la gestión de los stakeholders de la organización", en Horizonte, julio-agosto 1991, pp. 39-48.

Comisión Europea (2001). Libro Verde: Fomentar un marco europeo para la responsabilidad social. Comisión de las Comunidades Europeas. Disponibler en http://eur-lex.europa.eu/LexUriServ/site/ en/com/2001/com2001_0366en01.pdf. Consultado el 15 de octubre de 2012.

Cortina, A. (2000). Ética de la empresa. $5^{\text {a }}$ edición. Madrid:Trotta.
Friedman, Milton (1970). La responsabilidad social de las empresas es aumentar sus beneficios. Detroit: Teorías y Conceptos.

Gómez, A. B. (2009). Análisis organizacional basado en los lineamientos de la Responsabilidad Social Empresarial. Bogotá: Pontificia Universidad Javeriana.

González, M.d. (2005). La Responsabilidad Social Corporativa o responsabilidad social de la empresa. Bilbao: Eko Urriaren

Held, David y McGrew, Anthony (2003). Globalización/antiglobalización, Ed. Paidós.

Idowu, 0. y Filho, W. (2009). Prácticas globales de la Responsabilidad Social Empresarial. Madrid: Heidelberg.

Kant, E. (2000). Crítica de la razón práctica. Madrid: España. Alianza.

Neergaard, Peter (2006). Responsabilidad Social Corporativa. Alemania: Editores Académica. Traducción de Sergio Moral.

Núñez, Georgina (2003). La Responsabilidad Social Corporativa en un marco de desarrollo sostenible. CEPAL, Serie Medio Ambiente y Desarrollo.

Porter, M; Kramer, M. (2002). "La ventaja competitiva de la filantropía corporativa", en Harvard Business Review.

Porter, Michael (1985). "Competitive Advantage", en Harvard Business Review.

Valdivia, M. P. (2007). Análisis critico de los sistemas de medición de Responsabilidad Social Empresarial. Santiago de Chile: Uchile. 